\title{
Bilateral PICA Territory Infarcts of Right Lateral Medulla and Left Cerebellum via Spontaneous Common PICA Dissection
}

\author{
Kipyoung Jeon ${ }^{*}$ (D), Chonghwee Lee ${ }^{*}$ (D), Kijeong Lee ${ }^{\dagger}$ (D), Hyun Seok Choi ${ }^{\ddagger}$ (D), Bum-soo Kim ${ }^{\complement}$ (D), \\ Young Jin Ko" (D), Jaseong Koo* (D) \\ Department of Neurology, Seoul St. Mary's Hospital, The Catholic University of Korea*, Seoul; Department of Neurology, Eunpyeong St. Mary's \\ Hospital, The Catholic University of Korea ${ }^{\dagger}$, Seoul; Department of Radiology, Severance Hospital, Yonsei University College of Medicine ${ }^{\ddagger}$, Seoul; \\ Department of Radiology, Seoul St. Mary's Hospital, The Catholic University of Korea ${ }^{\complement}$, Seoul; Department of Rehabilitation Medicine, Seoul St. \\ Mary's Hospital, The Catholic University of Korea, Seoul", Korea
}

Bilateral cerebellar infarcts in posterior inferior cerebellar artery (PICA) territories have been previously reported and the common PICA (c-PICA) on one side was proposed as a possible mechanism. We report a case of bilateral PICA territory infarcts involving right lateral medulla and left cerebellum via spontaneous c-PICA dissection. Digital subtraction angiography (DSA) revealed that bilateral PICAs originated from the c-PICA arising from the right vertebral artery with luminal irregularity. Eccentric enhancement of the c-PICA on 3D multi-planar reformatted images suggested arterial dissection or atherosclerosis rather than vasculitis. Susceptibility-weighted image showed a blooming effect around the c-PICA and the phase map revealed eccentric high signal intensity on the vessel wall of the c-PICA, implicating intramural thrombus in c-PICA dissection. We investigated the mechanism and etiology of bilateral PICA territory infarcts using DSA and high-resolution magnetic resonance imaging (MRI) and elucidated the dissection of the c-PICA.

\section{J Neurosonol Neuroimag 2019;11(2):149-153}

Key Words: Cerebellar infarct; Lateral medullary infarct; Common PICA; Arterial dissection; High-resolution MRI
Received: June 12, 2019

Revised: July 24, 2019

Accepted: August 14, 2019

Address for correspondence: Jaseong Koo

Department of Neurology, Seoul St. Mary's Hospital, The Catholic University of Korea, 222 Banpo-daero, Seocho-gu, Seoul 06591, Korea

Tel: $+82-2-2258-6074$

Fax: +82-2-599-9686

E-mail:nrstroke@catholic. ac.kr
The posterior inferior cerebellar artery (PICA) supplies not only the caudal part of the cerebellar hemisphere and vermis, but also the dorsolateral portion of the medulla oblongata. ${ }^{1}$ Right and left PICAs typically originate from the right and left distal vertebral arteries, respectively. Bilateral cerebellar infarcts in PICA territories have been previously reported and the common PICA (c-PICA) on one side was proposed as a possible mechanism to explain bilateral PICA territory infarcts. ${ }^{2-4}$ Although autopsy-proven cases have supported this idea, ${ }^{5}$ there have been rare well-described case reports confirming the idea of c-PICA on one side and the underlying etiology using digital subtraction angiography (DSA) and high-resolution magnetic resonance imaging (MRI). Here, we report a case of bilateral
PICA territory infarcts involving right lateral medulla and left cerebellum via c-PICA dissection.

\section{CASE REPORT}

A 57-year-old female patient visited the emergency room (ER) due to acute sustained vertigo which started about 20 hours before she arrived ER during travel in Shanghai. She also complained of gait imbalance and nausea. Neurological examination revealed right ptosis, right upper and lower limb ataxia and gaze-evoked nystagmus, suggestive of Wallenberg's syndrome. Diffusion-weighted MRI confirmed right lateral medullary infarct and left cerebellar infarct in bilateral PICA 
territories. (Fig. 1A) Time of flight-magnetic resonance angiography (TOF-MRA) showed the right PICA arising from right vertebral artery, but not the left PICA. (Fig. 1B) We immediately administered a standard regimen comprising intravenous hydration, antiplatelet loading and high dose statins. On the 2nd hospital day, she started to express sustained stabbing headache in the right temporo-occipital region with right facial hypesthesia and dysphagia on neurological examination. Follow-up computed tomography (CT) showed neither edema progression nor hemorrhagic transformation on the left cerebellar infarct. On the 3rd hospital day, we initiated mannitol application to prevent brainstem compression resulting from edematous change of the left cerebellar infarct.

On the 4 th hospital day, we performed DSA exploring both the vertebral artery and PICA to check for the existence of a dissecting aneurysm causing subarachnoid hemorrhage as the patient experienced moderate-to-severe headache intermittently and also to investigate the mechanism underlying bilateral PICA territory infarcts. DSA revealed that bilateral PICAs originated from the c-PICA arising from the right vertebral artery. (Fig. 1C) 3D-reconstructed DSA showed luminal irregularity of the c-PICA without any fusiform aneurysm, implicating atherosclerosis or arterial dissection. (Fig. 1D) On the 5th hospital day, follow-up MRI confirmed that neither new infarction nor hemorrhagic transformation had occurred. (Fig. 1E, F) We tapered out the mannitol applied for prevention of edema progression on left cerebellar infarct. Transcranial Doppler sonography showed normal mean flow velocities and waveforms in
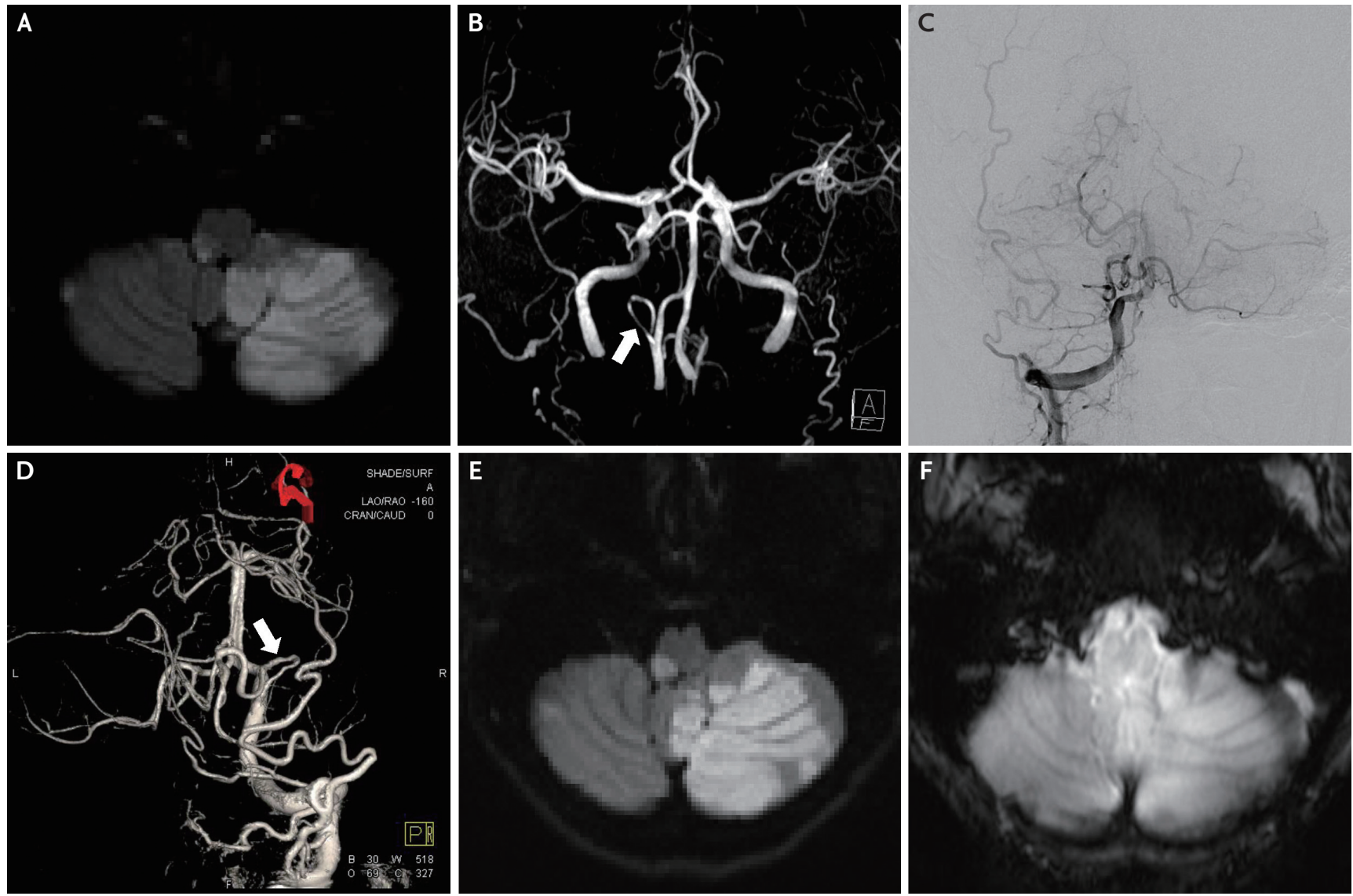

FIG. 1. (A) Diffusion-weighted magnetic resonance imaging (MRI) reveals the right lateral medulla infarct and left cerebellar infarct in bilateral posterior inferior cerebellar artery (PICA) territories. (B) Time of flight-magnetic resonance angiography (TOF-MRA) shows the right PICA arising from the right vertebral artery (white arrow), but not the left PICA. (C) Digital subtraction angiography (DSA) from the anterior view confirms that bilateral cerebellar PICA territories are supplied with blood via the common PICA (c-PICA) originating from the right vertebral artery. (D) 3 D-reconstructed DSA from the posterior view reveals luminal irregularity of the c-PICA without any definite dissecting aneurysm (white arrow).

(E) Diffusion-weighted MRI shows no infarct progression. (F) Gradient echo (GRE) image shows no hemorrhagic transformation. 
bilateral vertebral arteries.

Because she was young among patients with acute ischemic stroke and experienced sustained headache in the right occipital region, we were strongly suspicious of arterial dissection, particularly in the right vertebral artery or c-PICA. Eccentric enhancement of the c-PICA in $3 \mathrm{D}$ multi-planar reformatted (MPR) images supported atherosclerosis or arterial dissection rather than vasculitis. (Fig. 2A) Intriguingly, follow-up TOF-MRA on the 12th hospital day did not visualize the c-PICA, suggesting its flow disturbance. (Fig. 2B) There was no clinical or radiological progression of PICA infarction. Approximately 3 months later, follow-up TOF-MRA re-visualized the c-PICA. (Fig. $2 \mathrm{C}$ ) This flow variation in the c-PICA indicates arterial dissection rather than atherosclerosis. Susceptibility-weighted image (SWI) in a follow-up MRI showed a blooming effect around the c-PICA originating from the right vertebral artery. (Fig. 2D) Brain CT showed no calcification in the right vertebral artery or c-PICA. (Fig. $2 \mathrm{E}$ ) The phase map discovered eccentric high signal intensity on the vessel wall of the c-PICA, implicating intramural thrombus in the c-PICA dissection. (Fig. 2F) Interestingly, Fig. 2A, D and $\mathrm{F}$ showed the most prominent enhancement on $3 \mathrm{D}$ MPR, blooming effect in SWI and high signal intensity in the phase map which were performed on the 12th hospital day and corresponded to TOF-MRA in Fig. 2B. The $3 \mathrm{D}$ MPR, SWI and phase map corresponding to TOF-MRA in Fig. $1 \mathrm{~B}$ and Fig. $2 \mathrm{C}$ showed less prominent images. These serial high-resolution MRI and MRA findings also support arterial dissection rather than atherosclerosis.
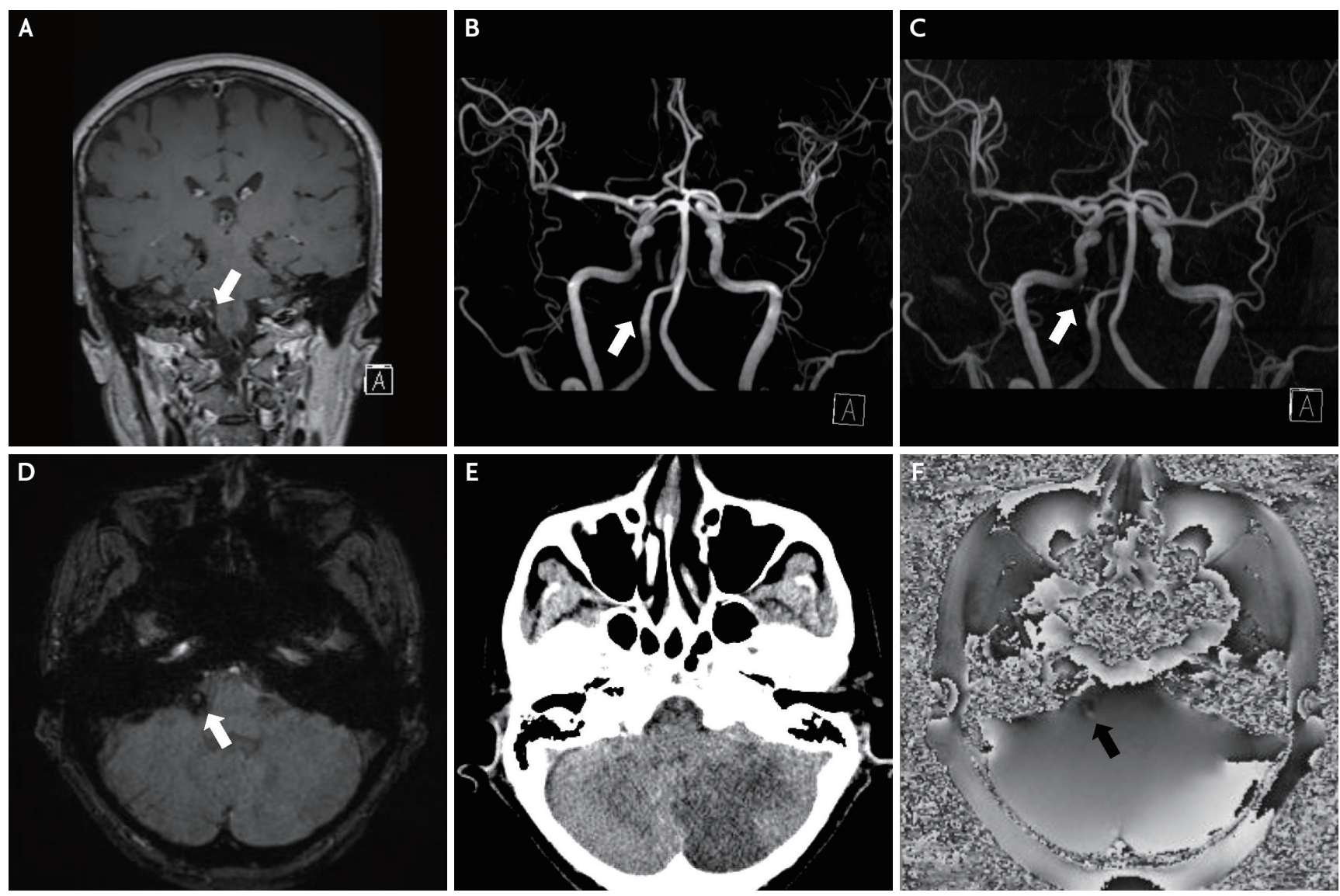

FIG. 2. (A) 3D multi-planar reformatted (MPR) images confirm eccentric enhancement of the c-PICA (white arrow). (B) Follow-up TOF-MRA fails to visualize the c-PICA (white arrow). (C) About 3 months later, follow-up TOF-MRA re-visualizes the c-PICA (white arrow). (D) Susceptibility-weighted image (SWI) in follow-up MRI shows a blooming effect around the c-PICA originating from the right vertebral artery (white arrow). (E) Brain computed tomography (CT) shows no calcification in the right vertebral artery and the c-PICA. (F) The phase map discovers eccentric high signal intensity on the vessel wall of the c-PICA (black arrow). 
She had no head or neck trauma history. The results of echocardiography and Holter monitoring revealed no source of cardioembolism and the level of serum D-dimer was unremarkable. We did not assess vasculitis or coagulopathy for a young-age stroke work-up because she was above 55 years of age. The patent foramen ovale (PFO) study yielded negative findings. She was transferred for gait and balance training.

\section{DISCUSSION}

Bilateral PICA territory infarcts affecting bilateral cerebellar regions have been previously reported. One case of bilateral PICA territory infarct involving the bilateral inferior cerebellum and right dorsolateral medulla has been reported. ${ }^{3}$ Our case described bilateral PICA territory infarcts involving right lateral medulla and left cerebellum. Several possible mechanisms of bilateral PICA territory infarcts have been proposed. The structural feature of the bilateral medial PICA branches arising from the c-PICA on one side was considered to cause bilateral PICA territory infarcts in the literatures. ${ }^{2-4}$ The most frequent cause of PICA infarcts has been documented as cardioembolism in Western countries. ${ }^{1,6}$ However, in East Asian countries, PICA territory infarcts were reported to be more associated with PICA or vertebral artery diseases than cardioembolism. ${ }^{7}$ PICA territory infarcts are thought to be equally divided between cardioembolism and posterior circulation arterial disease. ${ }^{8}$ Therefore, both the pathology of c-PICA and cardioembolism should be considered as the etiologies of bilateral PICA territory infarcts.

Etiologies of PICA territory infarcts include arterial dissections, especially in vertebral arteries or PICAs. Dissection of the vertebral artery is known to be a more frequent cause of PICA territory infarct than the PICA dissection. ${ }^{9}$ Spontaneous PICA dissections are rare, but often underestimated due to the difficulty in diagnosis. High-resolution MRI elucidated that the etiology of bilateral PICA territory infarcts in this case would be the dissection of the c-PICA rather than atherosclerotic or embolic occlusion. Unfortunately, we did not identify typical radiological findings suggesting arterial dissection in c-PICA such as pearl-and-string sign representing a dissecting fusiform aneurysm or tapered occlusion on DSA images.

T1-weighted MRI was previously recommended to elucidate intramural hematoma in arterial dissection particularly in the subacute stage. Unfortunately, T1-weighted MRI has frequently had difficulties in confirming small intramural hematoma in arterial dissection such as PICA dissections in the acute stage. Three serial sagittal T1-weighted images in this case failed to confirm hyperintense intramural signal in c-PICA dissection. Recent advances in high-resolution MRI have allowed frequent diagnosis of PICA dissections, but it remains difficult. Intracranial vessel wall imaging in high-resolution MRI studies has demonstrated eccentric enhancement in intracranial atherosclerosis or arterial dissection in contrast to concentric enhancement in vasculitis or Moyamoya disease. In this case, ${ }_{3} \mathrm{D}$ MPR images showed eccentric enhancement of the c-PICA, supporting arterial dissection or atherosclerosis rather than vasculitis. MRA did not show definite atherosclerotic burden in large arteries, but DSA confirmed luminal irregularity in the c-PICA. Follow-up TOFMRAs demonstrated variations in the visualization of the c-PICA, suggesting flow disturbance. These results support the possibility of the c-PICA dissection rather than atherosclerosis.

SWI was also considered to be effective in the diagnosis of PICA dissections as well as vertebral artery dissections. ${ }^{9,10}$ In this case, DSA did not show any fusiform aneurysm suggesting dissection in the c-PICA, but SWI revealed a blooming effect around the c-PICA originating from the right vertebral artery, indicating c-PICA dissection. The literature suggested that the eccentric high signal intensity in the phase map could correspond to intramural hematoma in the vertebral artery dissection. ${ }^{10}$ In this case, the phase map revealed eccentric high signal intensity in the vessel wall of the c-PICA, suggesting intramural hematoma in the c-PICA dissection.

Intriguingly, the right cerebellum and left lateral medulla in bilateral PICA territories remained unaffected in this case. We suppose that the left cerebellar and right lateral medullary infarcts in bilateral PICA territories could have been caused by artery-to-artery embolism from c-PICA dissection. DSA indicated that the right cerebellar PICA territory was supplied both by the branch arteries from c-PICA and by the collateral 
circulation from right superior cerebellar artery (SCA) and right anterior inferior cerebellar artery (AICA) other than the left cerebellar PICA territory. These findings may explain why the right cerebellar PICA territory was not infarcted in this case.

In conclusion, this case describes bilateral PICA territory infarcts involving the right lateral medulla and left cerebellum caused by spontaneous dissection of the c-PICA arising from the right vertebral artery. We investigated the mechanism and etiology of bilateral PICA territory infarcts using DSA and high-resolution MRI studies, and elucidated the dissection of the c-PICA. This case confirms the idea that bilateral PICA infarcts could be caused by the pathology of the c-PICA, especially arterial dissection in young patients.

\section{Conflicts of Interest}

No potential conflicts of interest relevant to this article was reported.

\section{REFERENCES}

1. Amarenco P. The spectrum of cerebellar infarctions. Neurology. 1991;41:973-979.

2. Tada Y, Mizutani T, Nishimura T, Tamura M, Mori N. Acute bilateral cerebellar infarction in the territory of the medial branches of posterior inferior cerebellar arteries. Stroke. 1994;25:686-688.
3. Sorenson EJ, Wijdicks EF, Thielen KR, Cheng TM. Acute bilateral infarcts of the posterior inferior cerebellar artery. J Neuroimaging. 1997;7:250-251.

4. Kang DW, Lee SH, Bae HJ, Han MH, Yoon BW, Roh JK. Acute bilateral cerebellar infarcts in the territory of posterior inferior cerebellar artery. Neurology. 2000;55:582-584.

5. Amarenco P, Hauw JJ, Henin D, Duyckaerts C, Roullet E, Laplane D, et al. Cerebellar infarction in the area of the posterior cerebellar artery. Clinicopathology of 28 cases. Rev Neurol (Paris). 1989;145:277-286.

6. Barth A, Bogousslavsky J, Regli F. The clinical and topographic spectrum of cerebellar infarcts: a clinical-magnetic resonance imaging correlation study. Ann Neurol. 1993;33:451-456.

7. Kim JS. Pure lateral medullary infarction: Clinical-radiological correlation of 130 acute, consecutive patients. Brain. 2003;126:1864-1872.

8. Kase CS, Norrving B, Levine SR, Babikian VL, Chodosh EH, Wolf PA, et al. Cerebellar infarction. Clinical and anatomic observations in 66 cases. Stroke. 1993;24:76-83.

9. Park MG, Choi JH, Yang TI, Oh SJ, Baik SK, Park KP. Spontaneous isolated posterior inferior cerebellar artery dissection: rare but underdiagnosed cause of ischemic stroke. J Stroke Cerebrovasc Dis. 2014;23:1865-1870.

10. Kim TW, Choi HS, Koo J, Jung SL, Ahn KJ, Kim BS, et al. Intramural hematoma detection by susceptibility-weighted imaging in intracranial vertebral artery dissection. Cerebrovasc Dis. 2013;36:292-298. 